\title{
Tumor renal benigno versus carcinoma renal: reporte de caso y revisión de la literatura
}

\section{Benign Kidney Tumor versus Renal Carcinoma: Case Report and Literature Review}

\author{
Jorge Enrique Salinas Perdomo ${ }^{1}$ Ángela Luna Meza² \\ ${ }^{1}$ Especialista en Hematología y Oncología Clínica, Oncología \\ Molecular, Clínica Medellín, Medellín, Colombia \\ 2 Asistente de Investigación, Departamento de Epidemiología Clínica \\ y Bioestadística, Facultad de Medicina, Pontificia Universidad \\ Javeriana, Bogotá, Colombia \\ ${ }^{3}$ Gerente de Proyectos, NeuroEconomix, Bogotá, Colombia \\ ${ }^{4}$ Departamento de Epidemiología Clínica y Bioestadística, Facultad de \\ Medicina, Pontificia Universidad Javeriana, Bogotá, Colombia
}

Camilo Castañeda-Cardona ${ }^{3}$ Diego Rosselli ${ }^{4}$

\author{
Address for correspondence Diego Rosselli, MD, MSc, Departamento \\ de Epidemiología Clínica y Bioestadística, Facultad de Medicina, \\ Pontificia Universidad, Javeriana, Carrera 7 No 40-62, Bogotá, \\ Colombia (e-mail: diego.rosselli@gmail.com).
}

\section{Resumen}

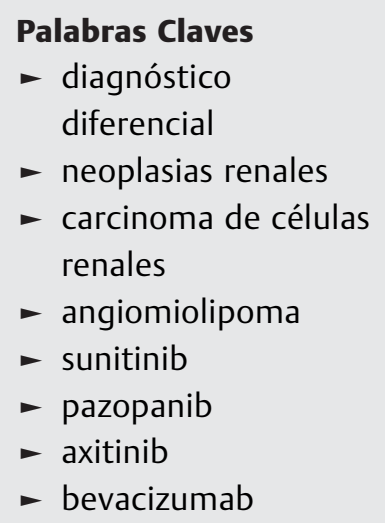

Introducción El cáncer renal representa 2,4\% de los casos diagnosticados de cáncer en la población general, es más común en hombres que en mujeres, y se presenta con más frecuencia entre la 6 ta y la 8 ta décadas de vida. Se estima que el $16 \%$ de los pacientes se diagnostican como enfermedad metastásica.

Objetivo Se presenta el caso de un paciente cuyo diagnóstico de carcinoma renal se confundió inicialmente con un tumor benigno.

Métodos A un hombre de 56 años de edad se le realizó hace 3 años ese diagnóstico en un estadio avanzado de la enfermedad, a pesar del hallazgo incidental de una masa, que se consideró benigna durante 5 años.

Resultados Al momento del diagnóstico de carcinoma de células claras, el tumor era Estadio IV, con metástasis a pulmón. Recibió primera línea de tratamiento con sunitinib, pero fue suspendido por toxicidad; segunda línea con pazopanib durante 1 año, después presentó progresión de la enfermedad, por lo cual se cambió a tratamiento con axitinib con respuesta parcial, sin embargo, se suspendió por toxicidad cardiaca, entre otras. Al momento el paciente ha recibido 5 ciclos de bevacizumab con adecuada tolerancia.

Conclusiones Es necesario resaltar la indicación de diagnóstico adecuado y manejo quirúrgico en masas renales sospechosas.

Introduction Kidney cancer represents $2.4 \%$ of diagnosed cases of cancer in the general population; it is more common in men than in women, and occurs more frequently between the 6 th and 8 th decades of life. It is estimated that $16 \%$ of patients are diagnosed as metastatic disease. received January 20, 2017 accepted September 13, 2017 published online May 29, 2018
DOI https://doi.org/ 10.1055/s-0038-1656553. ISSN 0120-789X. eISSN 2027-0119.
Copyright (c) 2019, Sociedad Colombiana License terms de Urología. Publicado por Thieme Revinter Publicações Ltda., Rio de Janeiro, Brazil. Todos los derechos reservados. 


\author{
Keywords \\ - diagnosis \\ - differential \\ - kidney neoplasms \\ - carcinoma \\ - renal cell \\ - angiomyolipoma \\ - sunitinib \\ - pazopanib \\ - axitinib \\ - bevacizumab
}

Objective To report the case of a male patient whose diagnosis of renal carcinoma was initially misdiagnosed as a benign tumor.

Methods We present a 56-year-old male diagnosed three years back with malignancy at an advanced stage of the disease, despite the incidental finding of a tumor that for 5 years was considered benign.

Results At the time of diagnosis of clear cell carcinoma, the tumor was Stage IV, with lung metastasis. He received first line treatment with sunitinib, which was discontinued due to toxicity. Subsequently, a second line with pazotinib for 1 year, then presented progression of the disease, so treatment was changed to axitinib with partial response., It was discontinued, however, due to cardiac toxicity, among others. At the time of writing, the patient has received 5 cycles of bevacizumab with adequate tolerance. Conclusions It is necessary to highlight the need for adequate diagnosis and surgical management in suspicious renal masses.

\section{Introducción}

El cáncer renal representa $2,4 \%$ de los casos diagnosticados de cáncer en la población general, es más común en hombres que en mujeres, y se presenta con más frecuencia entre la 6ta y la 8 ta décadas de vida. ${ }^{1}$ En Estados Unidos, se reportan 61 560 nuevos casos de cáncer renal, y 14 080, muertes durante 2015 . $^{2}$ En Colombia, se estima que entre 2007 y 2011 el cáncer de riñón tuvo una tasa de incidencia ajustada para la edad de 2,7 por 100.000 habitantes por año en hombres y 1,9 en mujeres. ${ }^{3}$ En los registros individuales de prestación de servicios de salud (RIPS) aparecen registrados, entre 2009 y 2013, 656 casos de tumores de riñón, pelvis renal y uréter en mujeres y 836 en hombres, lo que daría una tasa de incidencia acumulada, para el quinquenio, de 0,8 y 1,0 para hombres y mujeres, respectivamente. ${ }^{4}$

Contrario a lo que se pensaba hace unos años, hoy se conoce que el tamaño no es indicativo del comportamiento tumoral, y lesiones pequeñas pueden tener comportamiento maligno. $^{5}$

Las neoplasias renales pueden tener diferentes orígenes, pero $80 \%$ corresponden a carcinoma de células renales. Dentro de esos casos, el carcinoma de células claras representa un $81 \%$, seguido por carcinoma papilar, que es responsable de cerca del 14\% de los casos de cáncer de células renales. ${ }^{6}$ La diferenciación entre lesiones benignas y malignas, continúa siendo un reto diagnóstico para el cual es importante la sospecha clínica y la complementariedad con estudios imaginológicos. ${ }^{7,8}$

\section{Materiales y Métodos}

Presentamos a un paciente de 56 años de edad, con carcinoma renal de células claras, a quien el diagnóstico oficial se le realizó, tres años atrás, en un estadio avanzado de la enfermedad, previo seguimiento por una lesión aparentemente benigna. Previa autorización por parte del paciente, se realizó revisión de historia clínica, y revisión de la literatura con énfasis en las guías nacionales de manejo de cáncer renal ${ }^{9}$ y las guías americanas NCCN 2017. ${ }^{10}$

\section{Descripción del Caso}

A un hombre que actualmente tiene 56 años, procedente de Medellín, y sin antecedentes previos de importancia, se le realizaron en 2008 unos laboratorios de rutina solicitados por medicina general, y se encontró hematuria microscópica, por lo que se le solicitó valoración por Urología con sospecha de litiasis renal. Una ecografía renal reportó imagen hipoecoica, heterogénea, de contornos mal definidos, de $32 \times 34 \times 53 \mathrm{~mm}$ en el polo inferior del riñón derecho. La tomografía computarizada (TC) abdominal con contraste, mostró imágenes compatibles con hamartomas renales tipo angiomiolipomas (no se anota tamaño), así como un hemangioma en lóbulo derecho del hígado. Seis meses después se repitió la TC abdominal, que reportó imágenes en pedículo de riñón derecho compatible con teratoma, sin descartar malignidad. El servicio de Urología decidió continuar observación del paciente con controles semestrales hasta noviembre de 2013, cuando presentó dolor abdominal en hipocondrio y en flanco derecho, por lo cual se realizó resonancia magnética (RM) de abdomen, con medio de contraste, en donde se observó masa de $10 \times 8 \times 8,5 \mathrm{~cm}$, hipervascularizada con áreas de necrosis, sin componente graso, que comprometía la casi totalidad del parénquima renal, y que solo respetaba el aspecto posterior del polo superior, con disrupción de la cápsula en la región posterior, y que hacía contacto con el colon derecho en su aspecto anterior e inferior. Adicionalmente, un trombo tumoral ocupaba la vena renal, sin llegar al lumen de la vena cava inferior, y un hemangioma hepático en segmento VIII/V. También se realizó RM de tórax con medio de contraste que mostró incontables nódulos pulmonares con densidad de tejidos blandos de entre 5 y $310 \mathrm{~mm}$.

Dado que se trataba de un paciente con lesión de aspecto neoplásico renal derecho, con metástasis pulmonares, se llevó 
a nefrectomía radical en noviembre de 2013. El reporte anatomopatológico confirmó que se trataba de carcinoma de células claras, metastásico, pT3aN0M1, estadio IV. Fue valorado por el servicio de Oncología Clínica, con diagnóstico de carcinoma de células claras, estadio IV, con metástasis a pulmón, Fhurman 3, en muy buen estado general y funcional (ECOG 0), con índice de Karnofsky (IK) de 100\%, y un riesgo intermedio por escala del MSKCC (Memorial Sloan Kettering Cancer Center), candidato a inicio de tratamiento sistémico con intención paliativa con ITK, y sunitinib.

Entre diciembre de 2013 y junio de 2014, recibió la primera línea de tratamiento con sunitinib $50 \mathrm{mg}$ PO día, ciclos 4 semanas ON, 2 semanas OFF; uno de los tratamientos con indicación con nivel de evidencia $1 \mathrm{~A}$ como primera línea de tratamiento en las guías NCCN (National Comprehensive Cancer Network), para esa enfermedad. ${ }^{10}$ Se suspendió luego de 5 ciclos (a pesar de ajuste de dosis), por toxicidad gastrointestinal inaceptable dada por diarrea grado IV; adicionalmente presentó fatiga, náuseas e hiporexia grado II. En ese momento, se realizó revaloración con PETCT (tomografía por emisión de positrones), que mostró estabilidad de la enfermedad por criterios RECIST, con persistencia tumoral en retroperitoneo y pulmón (-Fig. 1).

En julio de 2014, dada la toxicidad inaceptable con la primera línea de terapia, se realizó cambio de tratamiento a pazopanib $800 \mathrm{mg}$ PO día, dosis que se redujo en septiembre del mismo año a $400 \mathrm{mg}$ PO día por diarrea grado III. En octubre de 2014 se realizó TC de tórax y abdomen

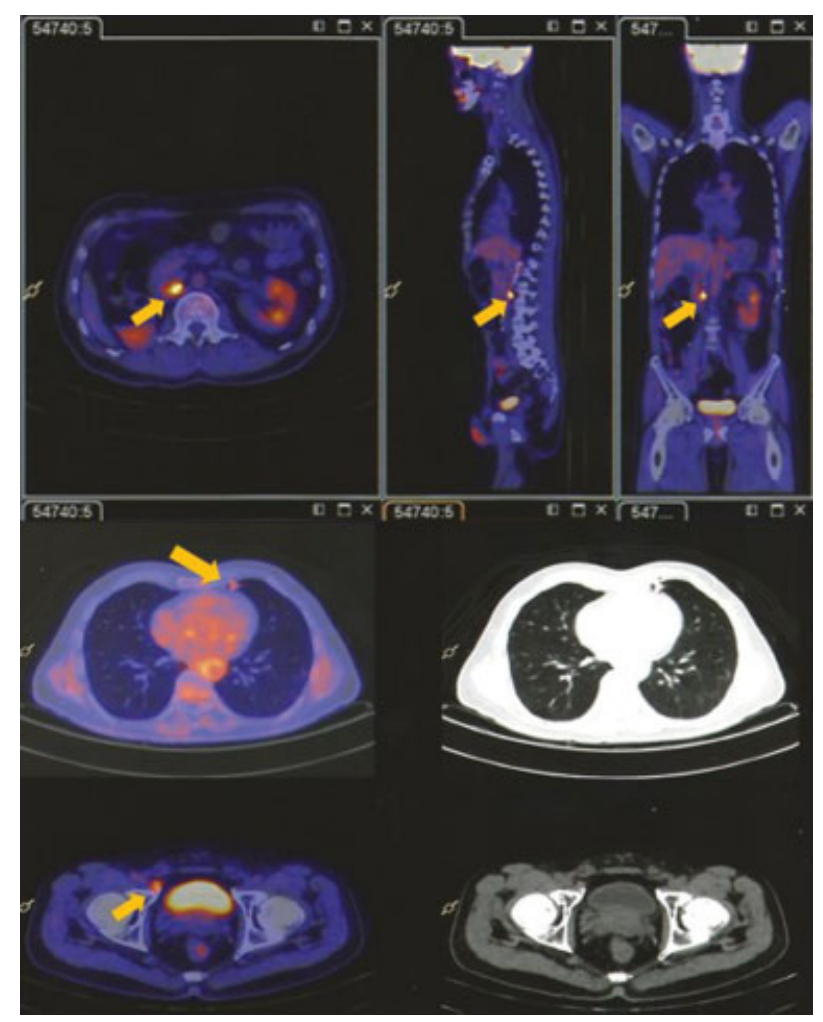

Fig. 1 Tomografía por emisión de positrones realizada en junio 2014; muestra persistencia tumoral en retroperitoneo y pulmón. contrastado con nefroprotección, y en él se observó respuesta completa en abdomen y estabilidad imaginológica en los nódulos pulmonares. Se consideró paciente con enfermedad estable y continuó el tratamiento instaurado.

En julio de 2015 el paciente presentó un episodio convulsivo, y se le realizó RM cerebral contrastada en la que se observaban múltiples lesiones metastásicas y edema cerebral. La revaloración sistémica con PETCT reveló además progresión en las lesiones nodulares pulmonares.

Por lo anterior se indicó realización de radioterapia holoencefálica (dosis 3000 cGy 10 fracciones/300 cGy) y tratamiento sistémico de tercera línea con axitinib $5 \mathrm{mg}$ PO c/12h, con dosis ascendente hasta $10 \mathrm{mg}$ PO c/12h, que se inició en agosto de 2015. La RM cerebral contrastada de control en octubre de 2015 mostró una lesión nodular cerebelosa derecha y otra anular cerebelosa izquierda, que realzaban con el gadolinio, con edema perilesional y a nivel supratentorial una lesión puntiforme en la segunda circunvolución frontal derecha que realza en forma nodular.

En abril de 2016, se realizó nuevamente RM cerebral contrastada que evidenció desaparición de las lesiones metastásicas, tanto frontal como cerebelosas, resolución del edema vasogénico y una pequeña área de cavitación en el hemisferio cerebeloso derecho. Esos hallazgos fueron interpretados como una respuesta completa en SNC, según los criterios RECIST (-Fig. 2), dados por la respuesta clínica favorable con desaparición de la cefalea, ganancia de peso, ausencia de déficit neurológico y estabilidad en su IK.

En mayo se realizó PETCT de control que mostró disminución importante de la actividad metabólica, número y tamaño de las lesiones nodulares secundarias al tumor primario en pulmón; pero con aumento del metabolismo focal en páncreas y tiroides, asociado a derrame pericárdico, sin compromiso hemodinámico ni posibilidad de pericardiocentesis. Lo anterior indicaba que el paciente presentaba una respuesta parcial al tratamiento, pero con hallazgos sugestivos de tiroiditis, pancreatitis y pericarditis, muy probablemente de origen farmacológico por la terapia antitumoral con axitinib.

El tratamiento con la tercera línea finalizó en junio de 2016. Esa terapia con axitinib, logró una buena respuesta de control tumoral (respuesta parcial por RECIST), pero generó toxicidad importante con hipotiroidismo grado II, diarrea grado IV, deterioro de la funcionalidad a un IK 60-70\%, pérdida de $8 \mathrm{~kg}$ de peso por hiporexia y cardiotoxicidad con pericarditis y disminución de la fracción de eyección ventricular izquierda (FEVI) a $36 \%$.

En julio de 2016 se inició cuarta línea de tratamiento sistémico con bevacizumab monoterapia, completando 5 ciclos mensuales de terapia cada 2 semanas. El paciente se encuentra actualmente asintomático, con recuperación de funcionalidad (IK 100\%) y ganancia de $4 \mathrm{~kg}$ de peso. Su control ecocardiográfico confirma la desaparición de la cardiotoxicidad con recuperación de la FEVI hasta la normalidad. Hasta la fecha (diciembre 2016), el paciente no ha presentado toxicidad y completa una sobrevida global en enfermedad metastásica de 36 meses. 

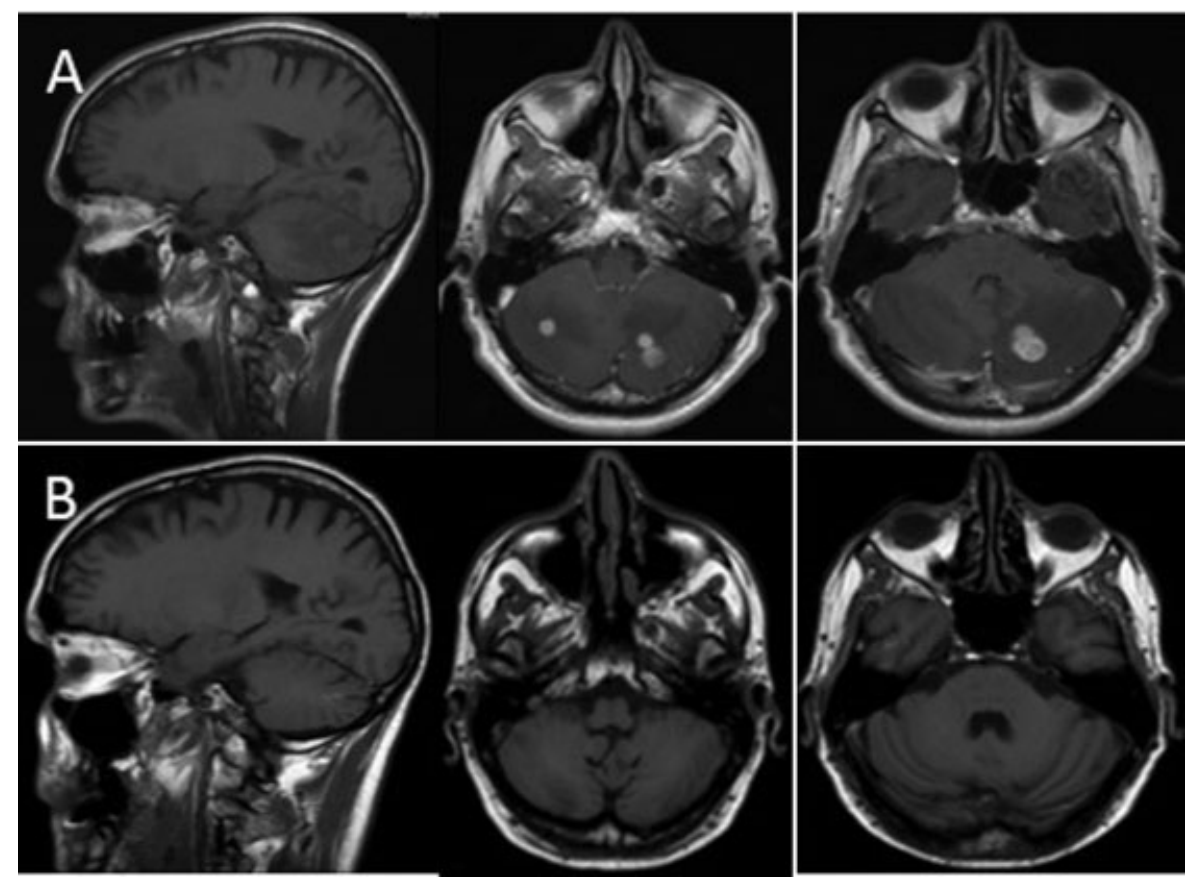

Fig. 2 Resonancias magnéticas de octubre 2015 (arriba) y abril 2016, que muestran desaparición de las lesiones metastásicas infratentoriales.

\section{Discusión}

Con mayor acceso a los servicios de salud y a estudios complementarios imagenológicos como la ecografía abdominal y la TC, ha aumentado el número de casos de neoplasias renales diagnosticadas en forma incidental en pacientes asintomáticos $\mathrm{u}$ oligosintomáticos. Ello ha permitido realizar la identificación de tumores renales malignos en estadios tempranos (I y II) hasta en un $85 \%$ de los casos, con un potencial curativo importante. ${ }^{11}$ Incluso, algunos estudios retrospectivos han relacionado el diagnóstico incidental versus el diagnóstico sintomático, con un beneficio en la sobrevida específica libre de cáncer a 5 años ( $100 \%$ versus $80 \%$ [ $p=0,001$ ] ) y la sobrevida libre de enfermedad a 5 años (98\% versus $62 \%$ [ $p<0,001]) .^{12}$

El diagnóstico diferencial de las masas renales sigue siendo un reto tanto para el radiólogo como para el clínico que evalúa esos pacientes. ${ }^{7}$ Un diagnóstico asertivo de una masa renal depende de varios factores críticos, como una buena historia clínica, la calidad y técnica de la imagen usada, la colaboración del paciente para la toma del estudio, la pericia del radiólogo y la exclusión de otras alteraciones que pueden simular una neoplasia renal. ${ }^{13}$

Desde el punto de vista radiológico es crucial el uso de contraste en el estudio de masas renales, para determinar la capacidad de captación y confirmar si es hiper o hipovascularizado. En principio, todas las lesiones malignas son hipervascularizadas, a excepción de las neoplasias papilares, ${ }^{14}$ por lo que la identificación de la captación del contraste, a través de la variación de la medición de las unidades Hounsfield (UH) cobra un papel singular. Una captación de $20 \mathrm{UH}$ o más es altamente sugestiva de realce al contraste. Sin embargo, aún no hay un consenso al respecto, sobre el valor específico que pueda ser usado en forma definitiva en el estudio de las masas renales. Algunos autores sugieren que valores superiores a 10 UH o entre $10-20 \mathrm{UH}$ son diagnósticas, ${ }^{15}$ mientras que otros consideran que la presencia de 15-20 UH no son conclusivas y requieren una caracterización imaginológica adicional, ${ }^{13}$ probablemente con RM.

En este caso, al paciente se le detectó en forma temprana un signo de alarma como es la hematuria microscópica, que llevó a la identificación de una masa renal derecha de $3 \times 4 \mathrm{~cm}$, sin metástasis, un estadio temprano con opción curativa. No hay una recomendación clara ni evidencia suficiente para sugerir la realización de biopsia renal a la mayoría de los pacientes con masas renales antes de la nefrectomía. Las guías americanas del NCCN 2017, lo dejan a consideración en presencia de lesiones pequeñas para establecer el diagnóstico de carcinoma renal y definir entre realizar seguimiento activo, criocirugía, ablación y/o radiofrecuencia. ${ }^{10}$ Por otro lado, los radiólogos consideran que la realización de biopsia para el estudio de masas renales indeterminadas, es injustificada en presencia de estudios imaginológicos de alta calidad, combinados con estudios de seguimiento en los casos indicados. ${ }^{13}$

Las Guías Colombianas de Urología para el manejo de carcinoma renal, publicadas en $2014,{ }^{9}$ destacan también la importancia del realce con el contraste en las masas renales para hacer la diferenciación entre lesiones benignas $\mathrm{y}$ malignas, además de recomendar el uso de RM contrastada en los casos indeterminados. La realización de resección quirúrgica de angiomiolipomas (como fue la consideración diagnóstica inicial en nuestro caso), con nefrectomía preservadora de nefronas si es posible, es sugerida en tres situaciones: ${ }^{9}$ Tumores mayores a $4 \mathrm{~cm}$, mujeres en edad fértil $\mathrm{y}$ pacientes en quienes no se puede asegurar el seguimiento adecuado (nivel de evidencia $3 \mathrm{~A}$ ). 
En el paciente hubo un claro problema en el seguimiento, con un control tomográfico que no descartaba malignidad sin asumir una conducta quirúrgica (nefrectomía). Una conducta expectante desde ese momento, sin la realización de una RM contrastada, fue riesgosa e inoportuna para el paciente. Desde el diagnóstico de la enfermedad metastásica en adelante, las conductas fueron acertadas, con una nefrectomía radical de citorreducción, manejo de primera línea y subsecuentes con base en los puntajes de riesgo y toxicidades, con una excelente respuesta al compromiso en SNC (lo cual es muy llamativo), logrando una sobrevida global hasta el momento de 36 meses.

\section{Conclusiones}

El estudio de las masas renales, requiere una correcta articulación entre los hallazgos clínicos e imaginológicos. El adecuado uso e interpretación de la TC contrastada en manos expertas, puede ser concluyente para el diagnóstico de una masa renal y su diferenciación entre benignidad y malignidad; sin embargo, en los casos indeterminados, el uso de la RM puede ser necesario. El papel de la biopsia renal continúa siendo controvertido y con el avance en las técnicas radiológicas, cada vez serán menos los pacientes candidatos a ese tipo de estudios invasivos y probablemente se relegaría a la necesidad de establecer la histología en aquellos pacientes no candidatos a nefrectomía.

\section{Responsabilidades Éticas}

Protección de personas y animales. Los autores declaran que para esta investigación no se han realizado experimentos en seres humanos ni en animales.

Confidencialidad de los datos. Los autores declaran que en este artículo no aparecen datos de pacientes.

Derecho a la privacidad y consentimiento informado. Los autores declaran que en este artículo no aparecen datos de pacientes.

\section{Nota}

Institución donde se llevó a cabo el trabajo: Clínica Medellín.
Conflicto de Intereses

Los autores declaran no tener conflicto de interés.

\section{Bibliografía}

1 National Comprehensive Cancer Network. (2016). NCCN clinical practice guidelines in oncology: kidney cancer (version 1.2017). pp. MS2

2 Siegel R, Miller K, Jemal A. Cancer Statistics 2015. CA Cancer J Clin 2015;65:6-7

3 Pardo C, Cendales R. Incidencia, mortalidad y prevalencia de cáncer en Colombia, 2007-2011. Primera Edición. Bogotá D.C. Instituto Nacional de Cancerología 2015, v.1.p.148

4 Alba LH, Alba M, Ortiz D, Otálora-Esteban M, Rosselli D. Análisis de los registros individuales de prestación de servicios de salud (RIPS) en cáncer en Colombia. Medicina (B Aires) 2016;38:223-231

5 Jayson M, Sanders $H$. Increased incidence of serendipitously discovered renal cell carcinoma. Urology 1998;51(02):203-205

6 Leibovich BC, Lohse CM, Crispen PL, et al. Histological subtype is an independent predictor of outcome for patients with renal cell carcinoma. J Urol 2010;183(04):1309-1315

7 Rumie Valois C, Ocampo J, Romero J. Avances en imágenes para la estadificación y seguimiento de pacientes con carcinoma de células renales. Urol Colomb. 2014;23:194-204

8 Kang SK, Huang WC, Pandharipande PV, Chandarana H. Solid renal masses: what the numbers tell us. AJR Am J Roentgenol 2014;202(06):1196-1206

9 Quiroga Matamoros W, Fernandez F, Citarella Otero D, Rangel J, Estrada Guerrero A, Patiño ID. Guía de manejo del carcinoma de células renales. Urol Colomb., (2016) pp. 169-189

10 National Comprehensive Cancer Network. (2016). NCCN clinical practice guidelines in oncology: kidney cancer (version 1.2017).p. MS3

11 Jain P, Surdas R, Aga P, et al. Renal cell carcinoma: Impact of mode of detection on its pathological characteristics. Indian J Urol 2009; 25(04):479-482

12 Dall'Oglio MF, Srougi M, Gonçalves PD, Leite K, Nesrallah L, Hering F. Incidental and symptomatic renal tumors: impact on patient survival. Sao Paulo Med J 2002;120(06):165-169

13 Israel GM, Bosniak MA. How I do it: evaluating renal masses. Radiology 2005;236(02):441-450

14 Vikram R, Ng CS, Tamboli P, et al. Papillary renal cell carcinoma: radiologic-pathologic correlation and spectrum of disease. Radiographics 2009;29(03):741-754, discussion 755-757

15 Chung EP, Herts BR, Linnell G, et al. Analysis of changes in attenuation of proven renal cysts on different scanning phases of triphasic MDCT. AJR Am J Roentgenol 2004;182(02):405-410 